\title{
Ueber den Verwandtschaftskreis der Empis stercorea Lin.
}

vom

Director H. Loew in Meseritz.

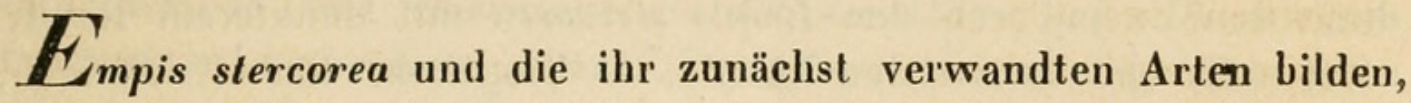
wie schon Meigen bemerkt hat, eine eigene Gruppe, welche sich durch die Verbindung einer Anzahl ziemlich auffallender Merkmale von allen anderen Gruppen der Empis-Arten bestimmt unterscheidet. Sie alle sind sehr kahle, vorherrschend rothgelb oder gelb gefärbte Arten mit langen, dünnen, bei beiden Geschlechtern einfachen und nur sehr kurz behaarten Beinen. Der Kopf derselben zeichnet sich durch gröfsere Entwickelung des Hinterkopfs aus und nimmt dadurch eine fast birnförmige Gestalt an, während das Collare zur Aufnahme desselben auffällig sattelförmig ausgebuchtet ist; die Augen des Männchens stofsen nicht zusammen, sondern sind, wie bei dem Weibchen, durch die verhältnifsmäfsig breite Stirn getrennt. Die Fühler sind verhältnifsmäfsig lang und schlank; das erste Glied derselben ist ansehnlich verlängert; das zweite Glied ist viel kürzer als das erste; das dritte Glied hat eine mehr oder weniger pfriemenförmige Gestalt. Die Linie, welche die Mittelstrieme des Thorax halbirt (ich will sie im Folgenden kurz die Mittellinie des Thorax nennen), ist unbehaart. Der Penis liegt mehr oder weniger frei und ist, besonders an seiner Wurzel, ziemlich dick. Die Flügel sind verhältnifsmäfsig ziemlich lang, aber nur mäfsig breit, bei beiden Geschlechtern von gleicher Bildung; das Geäder derselben ist bei allen Arten vollständig.

Mir sind bisher in Deutschland 10 Arten vorgekommen, welche in die Gruppe der Empis stercorea gehören, wenn man die Merkmale derselben so bestimmt, wie es im Vorhergehenden geschehen ist. Aufserdem kenne ich eine 11te Art, welche sich durch 
die Kürze des ersten Fühlergliedes von jenen 10 Arten zwar erheblich unterscheidet, in allen anderen plastischen Merkmalen aber vollständig mit denselben übereinstimmt. Ich bezweifle nicht, dafs es auch bei uns noch manche andere, in die Gruppe der Emp. stercorea gehörige Art geben wird, welche mir bisher nicht bekannt geworden ist, da die sehr grofse Häufigkeit einiger Arten ihnen ähnliche seltene Arten gar zu leicht übersehen läfst.

1) Die gemeinste von allen Arten ist die bereits von Linné beschriebene Empis stercorea. Sie ist durch die schwarze Färbung der Fühler, des Prothorasstigma und der Mittellinie des Thorax leicht kenntlich. Meigen's Emipis stigma halte ich für durchaus nichts weiter als eine häufig vorkommende Abänderung derselben mit dunklerem Randmale; wenigstens findet sich in Meigen's Beschreibung der Emp. stigma keine einzige Angabe, durch welche diese von Exemplaren der Empis stercorea mit dunklerem Randmale unterschieden werden könnle. - Umgekehrt finden sich auch Exemplare der Emp. stercorea, bei denen das Randmal fehlt.

Mit Emp. slercorea pflegen zwei ihr sehr ähuliche Arten verwechselt zu werden, die auf eine der publicirten Arten zurückzuführen mir nicht gelungen ist. Ich nenne sie Emp. dimidiata und univitlala. - Beide stimmen in der Färbung und in der Zeichnung nahebei mit Emp. stercorea überein, auch sind die Fühler derselben ganz von demselben Baue wie bei Emp. stercorea und, wie bei dieser, ganz schwarz. Beide unterscheiden sich dagegen von Emp. stercorea schon dadurch sehr leicht, dafs bei ihnen das Prothoraxstigma keine dunkle Einfassung hat, während es bei Emp. stercorea stets schwarz eingefalst ist.

2) Emp. dimidiata kommt der Emp. stercorea in der Gröfse gleich oder doch nahebei gleich; der Kopf ist wie bei dieser gefärbt; die schwarze Mittellinie des Thorax ist mindestens ebenso schmal und, wie die ganze Oberseite des Thorax, matter; auch pflegt dieselbe an ihrem Vorderende etwas crweitert zu sein. Schildchen und Hinterrücken sind einfarbig rothgelb, während bei Emp. stercorea sich auf dem Schildchen fast immer, auf dem Hinterrücken immer ein schwarzer Fleck findet; die Flügeladern sind weniger dunkel als bei Emp. stercorea gefärbt und erscheinen deshalb schwächer; ein deutliches Randmal ist bei keinem meiner Exemplare vorhanden, während er sich bei Emp. stercorea in der Regel findet. Die seitlichen Lamellen des Hypopygium sind breiter und am Ende viel stumpfer.

3) Emp. univittata, welehe etwas kleiner als Emp. ster- 
corea ist, wird von Zetterstedt Dipt. Scand. I. p. 378 als var. $b$. der letzteren erwähnt. Die schwarze Färbung hat am Hinterkopfe eine viel gröfsere Ausdehnung als bei Emp. stercorea. Auf dem Thoraxrücken ist nicht nur die Mittelllnie, sondern die ganze Mittelstrieme sammt der Mittellinie schwarz gefärbt, so dafs die dadurch gebildete durchgehende schwarze Längsbinde gut doppelt so breit als bei Emp. slercorea ist; betrachtet man diese Binde ganz von vorn her, so erscheint sie bei gut conservirten Exemplaren nur an den Seiten schwarz, während sich auf der Mitte derselben eine matte, schwarzbraune, von grauweifslicher Bestäubung umgebene Mittellinie zeigt. Fast die ganze Oberseite des Schildchens und die Mitte des Hinterrückens sind schwarzbraun. Die dunkle Mittelstrieme des Hinterleibs pflegt breiter als bei Emp. siercorea zu sein. Die Flügeladern sind auch bei dieser Art erheblich heller als bei Emp. sterocea und erscheinen deshalb schwächer; ein deutliches Randmal ist bei keinem meiner Exemplaren vorhanden. Das Hypopygium ist dem der Emp. stercorea ähnlich, doch sind die seitlichen Lamellen desselben etwas schmäler und erheblich spitziger, auch an der äufsersten Spitze deullich geschwärzt; die obere Lamelle aber ist nicht nur am Oberrande intensiver geschwärzt und dichter mit kurzen, schwarzen Härchen besetzt, sondern sie hat auch jederseits einen kleinen schmalen, ebenso gefärbten und behaarten Anhang, von dem bei Emp. stercorea keine Spur vorhanden ist.

4) Ziemliche Aehnlichkeit mit Emp. univittata hat eine noch etwas kleinere Art, welche ich Emp. aequalis nenne. Sie unterscheidet sich von jener leicht dadurch, dafs nicht nur Mittelstrieme und Mittellinie des Thorax, sondern auch die Interstitien zwischen Mittelstriemen und Seitenstriemen schwarz gefärbt sind, so dafs bei ihr die den Interstitien angehörigen Borstenreihen noch auf der schwarzen Längsbinde, nicht wie bei Emp. univittata ausserhalb derselben, stehen. Andere sehr in die Augen fallende Unterschiede sind die viel geringere Länge des dritten Fühlerglieds und die schärfere Begrenzung und viel geringere Ausdehnung der schwarzen Färbung am Hinterkopfe bei Emp. aequalis. Auch sind bei Emp. aequalis das ganze Schildchen einschliefslich seines Randes und der Hinterrücken der Breite des Schildchens entsprechend braunschwarz gefärbt. Die dunkle Mittelstrieme des Hinterleibs pflegt sich noch viel mehr als bei Emp. univittata auszubreiten und erstreckt sich, besonders bei dem Weibchen, oft bis in die Nähe des Seitenrandes. Die Flügel pflegen noch etwas weniger als bei Emp. univittata getrübt zu sein und haben, wie bei dieser, kein deutli- 
ches Randmal. - Auch mit Emp. bilineata m., von der sogleich ausführlicher die Rede sein soll, hat Emp. aequalis ziemliche Aehnlichkeit; namentlich stimmt sie im Baue der Fühler und in der Färbung und Zeichnung des Kopfes gut mit ihr überein, nur ist der sehwarze Fleck des Hinterkopfs bei ihr minder breit. Man unterscheidet sie von derselben sehr leicht an der geringeren Gröfse, welche kaum die der Emp. lutea übertrifft, und an den borstenartigen Haaren auf den Interstitien zwischen Mittelstrieme und Seitenstriemen des Thorax, welche bei ihr von gewöhnlicher Länge, bei Emp. bilineata aber von ganz aufserordentlicher Kürze sind. Aufserdem ist bei Emp. aequalis die dunkle Längsbinde des Thorax schmäler und schärfer begrengt, da sie mit den Interstitien abschneidet, während sie bei Emp. bilineata sich erst etwas jenseits derselben allmälig abschattirt; auch ersčheint sie schwärzer und nicht so vollkommen matt, weil sie von weniger dichter und weniger in das Gelbe ziehender Bestäubung bedeckt ist; endlich bilden die Interstitien bei Emp. aequalis nur wenn man den Thorax ganz von vorn her betrachtet, schwärzere, fast etwas glänzende, aber nur undeutlich begrenzte Streifen, während sie bei Emp. bilineata zwei, in jeder Ansicht vollkommen deutliche, scharfbegrenzle, schwarzbraune, vollständig matte, von einem bis zum anderen Ende des Thorax gehende Streifen bilden. - Das Hypopygium ähnelt dem des Männchens von Emp. bilineata, doch ist die obere Lamelle etwas gröfser.

5) Diejenige Art, welche ich Emp. bilineat a nenne, ist zuerst von Fabricius im Syst. Antl. als Emp. punctala kenntlich beschrieben worden, aber keineswegs, wie er angenommen hat, mit der von Meigen früher in der Klassifikation bekannt gemachten Emp. punctata identisch. Meigen hat diesen Umstand bei der Untersuchung der Fabricius'schen Sammlung festgestellt und sich durch denselben bestimmen lassen, im 6ten Theile seines Hauptwerks seiner eigenen Emp. punctata den Namen Emp. ignota beizulegen und die Fabricius'sche Art als Emp. punctata zu beschreiben, während dieser Name doch nothwendig derjenigen Art verbleiben mufs, welcher er zuerst von Meigen beigelegt worden ist. Zetterstedt beschreibt gegenwärtige Art als Emp. Lestacea Fabr., was meines Wissens zuerst von Staeger bemerkt worden ist. Die Beschreibung, welche Fabricius von seiner Emp. tesıacea giebt, kann unmöglich auf gegenwärtige Art bezogen werden, dagegen kann sie recht wohl auf die Vermuthung führen, dafs er bei Abfassung derselben die Emp. punctata der Meigen'schen Klassifikation vor sich gehabt habe; ich würde dies für äufserst wahrscheinlich halten, wenn er nur bei 
ihrer Vergleichung mit $\boldsymbol{E} \boldsymbol{m}$. stercorea nicht sagte: forte ejus varietas paulo major, während Emp. punctata Meig. K1. doch etwas kleiner als Emp. stercorea ist. - Der Kopf der Emp. bilineata ist gelb mit einem sehr grofsen schwarzen Flecke auf dem Hinterkopfe; die Fühler sind schwarz, das dritte Glied derselben minder lang als bei Emp. stercorea. Auf den Interstitien zwischen den Seitenstriemen und der Mittelstrieme des Thorax liegen zwei durchgehende, scharfbegrenzte Streifen von völlig matter, schwarzbrauner Färbung, welche in jeder Richlung gleich sehr in die Augen fallend sind; zwischen diesen Streifen zeigt der Thoraxrücken eine völlig matte, gelblich aschgraue Färbung ohne Spur einer anders gefärbIen Mittellinie; dieselbe Färbung säumt die Aufsenseite jedes dieser Streifen; sie rührt von dichter gelblicher Bestäubung auf schwärzlichem Grunde her. Die auf den Interstitien befindlichen borstenartigen Härchen sind äufserst wenig zahlreich und so aufserordentlich kurz, dafs sie nur schwer wahrzunehmen sind, was bei keiner anderen mir bekannten Art der Fall ist. Das Schildchen mit Ausnahme seines Randes und der grölste Theil des Hinterrückens sind schwärzlichgrau gefärbt. Die schwarze Mittelstrieme des Hinterleibs ist ziemlich breit; schwarze Seitenstriemen, wie sie sich bei Emp. slercorea, dimidiata und univittata finden, sind bei ihr, wie bei Emp. aequalis, in der Regel gar nicht vorhanden. Die seitlichen Lamellen des Hypogygiums sind verhältnifsmäfsig grofs und mehr als bei den meisten anderen Arten nach oben gerichtet; sie haben einen sehr langen, geraden Endrand, dessen Unterecke zipfelförmig verlängert ist; die lange Behaarung derselben ist blafs; die einfache obere Lamelle ist klein und ziemlich versteckt, an ihrem Oberrande nur etwas gebräunt.

6) Die Art, welche Meigen in der Klassifikation und im 3ten Theile der system. Beschreibung als, Emp. punctata beschreibt, und der er dann im 6 en Theile den Namen Emp. ignota beilegt, kann nur entweder, wie ich es thue, auf diejenige Art, welche 'Letterstedt in den Dipt. Scand. rccht kenntlich als Emp. ignota beschrieben hat, oder auf die demnächst zu besprechende Art, welche ich Emp. semicinerea nenne, gedeutet worden. Letzteres zu thun verbietet sich dadurch, dafs 1 ) bei Emp. semicinerea nur die Interstitien zwischen der Mittelstrieme und den Seitenstriemen des Thorax, nicht aber die Mittellinie dunkel gefärbt ist, während Meigen doch von drei dunklen Thorasstriemen spricht, 2) dafs Emp. semicinerea, soviel bekannt ist, eine ausschliefslich im Mittelgebirge vorkommende Art ist, während Emp. punctata nach Meigen's An- 
gabe eine bei Stollberg häufige Art sein soll. Für die Deutung, welche Zetterstedt der Meigen'schen Beschreibung giebt, spricht dagegen die äufserst grofse Gemeinheit dieser Art im ganzen nördlichen Deutschland, in Verbindung mit dem Umstande, dafs keine andere Meigen'sche Beschreibung auf dieselbe pafst; auch trifft die von Meigen gegebene Beschreibung des Thoraxrückens gut zu. Bedenken gegen diese Deutung können allenfalls dadurch erregt werden, dafs Meigen den Thorax schlechthin aschgrau nennt, ohne die gelbe Färbung der Brustseiten zu erwähnen, und dafs er sowohl in der Klassifikation wie im 3ten Theile des Hauptwerks die Gröfse derselben gleich der von Emp. stercorea angiebt, während dieselbe doch etwas geringer ist. Da Meigen auch bei der im 6ten Theile beschriebenen Emp. punclata Fabr. in der Diagnose den Thorax schlechthin grau nennt, und erst in der Beschreibung der gelben Farbe der Brustseiten beiläufig gedenkt, so scheint mir auf den ersten Umstand nicht gar zu viel Gewicht zu legen. Darf ich annehmen, dafs Wiedemann bei der Abfassung der im 3ten Bande von Meigen's Werk mitgetheilten Beschreibung der Emp. trigramma über die Bestimmung der wahren Emp. punctata Meig. im Sicheren gewesen sei, so bin ich bei der Art, in welcher er sich über deren Aehnlichkeit mit Emp. punctata ausspricht, fast gezwungen das Vorhandensein von gelbgefärbten Brustseiten bei Emp. punctata Meig. anzunehmen, und finde dann das, was er über die unbestimmtere Begrenzung der Thoraxstriemen von Emp. punctata sagt, für diese sehr charakteristisch. Schliefslich bemerke ich, dafs für die Art, welche ich mit Zetlerstedt für die Meigen'sche Emp. punctata halte, die bereits von Zetterstedt bemerkte Anwesenheit ansehnlicher grauer Flecke über den Mittel- und Hinterhüften ein ganz brauchbares Merkmal ist.

7) Emp. semicinerea m., welche hinsichtlich der plastischen Merkmale ganz und gar in den Kreis gegenwärtiger Gruppe gehört, unterscheidet sich von allen anderen mir bekannten Arten derselben äufserst leicht dadurch, dafs die Grundfarbe von Kopf und Thorax schwärzlich ist, so dafs diese Körpertheile in Folge ihrer weifslichen Bestäubung grau erscheinen. Am Kopfe pflegt nur der seitliche Mundrand eine schmale gelbe Einfassung zu haben. Die Fühler sind schwarz. Auf dem Thoraxrücken haben die Interstitien zwischen Mittel- und Seitenstrienıen eine völlig matte, schwarzbraune Färbung, welche indessen nur so weit wie die Seitenstriemen, aber nicht wie bei Emp. bilineata vom Vorderrande bis zum Hinterrande des Thorax reicht; die borstenartigen Haare auf den 
Interstitien sind von der gewöhnlichen Länge und Stärke; von einer dunkeln Mittellinie ist bei den meisten Exemplaren nichts zu sehen, oder sie wird doch nur wahrgenommen, wenn man die Oberseite des Thorax ganz von hinten her betrachtet und zeigt dann keine braune, sondern eine schwarze Färbung. Schildchen und Hinterrücken sind mit dem übrigen Thorax gleichgefärbt. Der gelbe Hinterleib zeigt, wie bei Emp. slercorea, dimidiata, univittata und punctata, drei schwarze Striemen, deren mittelste von ziemlicher Breite ist; nicht selten sind auch die Hinterränder der einzelnen Abschnitte schwarzbraun gefärbt. Hüften und Beine sind gelb; die Spitze der Schienen und die Füfse sind gebräunt oder geschwärzt. Flügel mit mäfsig bräunlicher Trübung wie bei Emp. punctata, ohne deutliches Randmal. Die seitlichen Lamellen des Hypopygiums sind von mäfsiger Grölse, stumpf und nur mit kurzer Behaarung besetzt; die obere Lamelle ist einfach und an ihrem Oberrande gebräunt.

8) Emp. trigramma Meig. ist an ihren 3 breiten, durch gelbe Interstitien getrennten Thoraxstriemen und an dem einfachen Baue des Hypopygiums sehr kenntlich; die seitlichen Lamellen des letzteren sind schräg nach oben gerichtet und schliefsen sich der zurückgeschlagenen, verhältnifsmäfsig grofsen Oberlamelle eng an. In Meigen's Werke ist die Vermuthung ausgesprochen, dafs das dort beschriebene Exemplare der Hoffmannsegg'schen Sammlung wohl aus Portugal herstammen möge; die Häufigkeit der Art bei uns und der Umstand, dafs seitdem nichts weiter über ein so südliches Vorkommen derselben bekannt geworden ist, machen es wahrscheinlicher, dafs das in Meigen's Werke beschriebene Exemplar aus der Berliliner Gegend stammen möge. Der Umsland, dafs für Emp. trigramma nur eine Körperlänge von $3 \frac{1}{3}$ Lin. angegeben ist, scheint mir kaum darauf hinzudeuten, dafs sich die Beschreibung nicht auf unsere, sondern auf eine etwas kleinere südeuropäische Art beziehe; es ist ja genügend bekannt, was für ein trügerisches Merkmal die Angabe über die Körperlänge ist, wenn dieselbe nach trocknen Exemplaren ohne Rücksicht auf das bei dem Trockenwerden erfolgte Einschrumpfen bestimmt wird.

9) Empis lutea Meig. ist nicht wohl zu verkennen, da nur die Fühler, ein die Ocellen umgebender Punkt und der Rand der oberen Lamelle des Hypopygiums schwarz, aufserdem die Füfse in ziemlicher Ausdehnung geschwärzt sind. Der Bau des Hypopygiums ist ein äufserst auffallender, da die seitlichen Lamellen desselben in einen langen, fast griffelförmigen, mit langen schwarzen Haaren beBerl. Entomol. Zeitschr. XI. 
setzten Fortsatz verlängert sind. Das Weibchen ist auf der Mitte der hinteren Thoraxhälfte mehr beborstet, als das irgend einer andern der hierher gehörigen Arten, und unterscheidet sich dadurch von seinem Männchen in einer Weise, die leicht ungerechtfertigte Zweifel über die Zusammengehörigkeit beider erwecken kann.

10) Die kleinste aller mir bekannten Arten aus gegenwärtigem Verwandtschaftskreise nenne ich Emp. nana. Sie ist, wie Emp. lutea, ganz gelb gefärbt und dieser überhaupt sehr ähnlich, doch erreicht sie die Gröfse, welche die allerkleinsten Stücke dieser haben, noch nicht. Die Fühler sind wie bei Emp. lutea schwarz und die Füfse gewöhnlich bis gegen die Wurzel hin schwarzbraun; auch die Flügel sind von derselben verdünnt braungelblichen Färbung und ohne deutliches Randmal, wie bei dieser. Beide Geschlechter scheinen sich von der Emp. lutea stets durch die ziemlich schwärzliche, aber weifslich bestäubte Stirn zu unterscheiden. Das Männchen ist von dem der $\boldsymbol{E m p}$. lutea sehr leicht an dem ganz verschiedenen Baue des Hypopygiums zu unterscheiden; der Endrand der seitlichen Lamellen desselben ist seiner ganzen Länge nach mit borstenartigen schwarzen Haaren besetzt und hat auf seiner Mitte eine Bucht, so dafs sich ein ganz abgerundeter oberer und ein längerer, ziemlich spitziger unterer Lappen unterscheiden läfst; die obere Lamelle ist klein und fast bis zu ihrem Grunde in zwei linienförmige, schwarze und mit schwarzen Härchen besetzte $Z_{w e i g e}$ gespalten. Ob sich das Weibchen vom Männchen durch stärkere Behaarung der Mitte der hinteren Thorashälfte unterscheidet, wie dies bei Emp. lutea der Fall ist, vermag ich nicht zu sagen, da ich kein Weibchen besitze, bei dem der Nadelstich eine sichere Wahrnehmung zuläfst.

11) Nach Besprechung der 10, in jedem Falle zur Gruppe der Emp. stercorea gehörigen deutschen Arten, welche mir bisher bekannt geworden sind, hal,e ich noch einige Worte über die oben erwähnte elfte Art hinzuzufügen, welche sich von jenen durch den Fühlerbau nicht unerheblich unterscheidet, in allen übrigen hier in Betracht kommenden Merkmalen aber mit denselben übereinstimmt. Ich halte dieselbe für Emp. parvula Egg. Sie gleicht in der Körperfärbung und Zeichnung bei weitem an meisten der Emp. dimidiata, doch ist die schwarze Mittellinie des Thorax noch schmäler und zuweilen sehr undeutlich; auch ist das Prothoraxstigma stets braunschwarz gesäumt, während es bei Emp. dimidiata mit den Brustseiten gleichgefärbt ist. Die beiden ersten Fühlerglieder sind rothgelb, das erste kaum etwas länger als das kurze zweite; das 
dritte Fühlerglied, welches mit Ausnahme seiner Basis schwarz gefärbt ist, hat etwa die Form und die verhältnifsmäfsige Gröfse, wie bei Emp. aequalis und bilineala. Das Schildchen pflegt mit dem Thorax gleichgefärbt zu sein; die Mitte des Hinterrückens hat einen schwarzen Fleck. Die lehmgelbliche Trübung der Flügel ist äufserst verdünnt und ein deutliches Randmal nicht vorhanden. Will man Emp. parvula zur Gruppe der Emp. stercorea bringen, so darf unter den oben angegebenen Merkmalen dieser nur die Verlängerung des ersten Fühlerglieds gestrichen werden.

Die Diagnosen der besprochenen Arten können etwa in folgender Weise festgestellt werden. Ich darf wohl nicht erst daran erinnern, dafs die Flügellänge einen richtigeren Maafsstab für die verhältnifsmäfsige Gröfse der einzelnen Arten giebt, als die so schwer richtig zu bestimmende Körperlänge, und dafs die untere Grenze der letzteren nur von den Männchen, die obere nur von den Weibchen erreicht wird. Von den Synonymen habe ich nur die wichtigeren angeführt.

1. Emp. stercorea Linn. $\sigma^{7}$ et ㅇ. Flava, antennis, maculá occipitali, stigmate prothoracis thoracisque lineâ mediâ nigris, stigmate alarum plerumque conspicuo. Hypopygii lamellae laterales modice latae, acutae, lamella supera simplex, marginis summi anguste nigro-limbati angulis singulis in lobum perbrevem atrum dilatatis. - Long. corp. $2 \frac{2}{3}-3 \frac{3}{4}$ lin., Long. al. $3 \frac{7}{12}-4 \frac{1}{2}$ lin.

Syn. Empis stercorea Linn. Faun. Suec. II. 1899.

Empis stercorea Fall. Emp. 18. 5.

Empis stercorea M eig. Syst. Beschr. III. 36. 38.

Empis stercorea Zett. Dipt. Scand. I. 377. 10. (exclusâ tamen var. b.)

Empis stigma M eig. VII. 88. 70.

Hab. Europa media et septentrionalis.

2. Emp. dimidiata m. $\delta$ et 9 . Flava, stigmate prothoracis scutelloque concoloribus, antennis, macula occipitali thoracisque linea mediâ nigris. Hypopygii lamellae laterales latae, obtusae; lamella supera simplex, marginis summi anguste nigro-limbati angulis singulis in lobum perbrevem atrum dilatatis. - Long. $2 \frac{3}{4}-3 \frac{2}{3}$ lin., Long. al. $3 \frac{5}{12}-3 \frac{1}{1} \frac{1}{2}$ lin.

Hab. Germania australis. 
3. Emp. univittata m. ठ et + . Flava, stigmate prothoracis concolore, antennis, maximâ occipitis parte et thoracis vittâ mediâ nigris. Hypopygii lamellae laterales modice latae, acutae, summo apice nigro; lamellae superae margo summus niger, confertissime nigro-pilosulus, ex utroque angulo apophysim linearem nigram emittens. - Long. corp. $2 \frac{1}{6}-2 \frac{1}{2}$ lin., Long. al. $2 \frac{5}{6}-3 \frac{1}{2}$ lin.

Syn. Empis stercorea var. b. Zett. Dipt. Scand. I. 388.

Hab. Europa media et septentrionalis.

4. Emp. aequalis m. ठ et ㅇ․ Flava, stigmate prothoracis concolore, antennis, maculâ occipilali thoracisque viltâ mediâ cum interstitiis, quibus a vittis lateralibus distinguitur, nigris. Hypopygium pallide-pilosum; lamellae laterales angustae, infero marginis apicalis angulo longissime producto; lamella supera simplex, margine nigro nullo. - Long. corp. $2 \frac{1}{1 \frac{1}{2}}-2 \frac{1}{2}$ lin., Long. al. $2 \frac{3}{4}-$ $2 \frac{1}{1} \frac{1}{2} \operatorname{lin}$.

Hab. Germania.

5. Emp. bilineata m. đ’ et + . Flava, antennis maculâque occipitali nigris, thoracis dorso opaco, vittâ mediâ vitlarumque lateralium marginibus interioribus cinereis, vittarum interstitiis nigro-fuscis, pilos perpaucos, omnium brevissimos gerentibus. Hypopygium pallide pilosum; lamellae laterales modice lalae, marginis apicalis angulo infero longissime producto; lamella supera simplex, margine nigro nullo. - Long. corp. $2 \frac{2}{3}-3 \frac{5}{12}$ lin., Long. al. $3 \frac{7}{\frac{7}{2}}-$ $3 \frac{3}{4}$ lin.

Syn. Empis punctata Fabr. Syst. Antl. 142. 22.

Empis punctata Meig. Syst. Beschr. VI. 338. 51.

Empis testacea Z ett. Dipt. Scand. I. 378. 11. (exclusis tamen synonymis).

Hab. Europa media et septentrionalis.

6. Emp. punctata Meig., Kl. $ఠ$ et ㅇ. Flava, antennis nigris, fronte et occipile fere toto nigricantibus, thoracis dorso praeter limbos laterales obscure cinereo, subobsolete nigro-viltato, pleuris prope coxas posteriores cinereo-maculatis. Hypopygii lamellae laterales acutae, extremo apice nigro et nigro-piloso; lamellae superae margo summus nigro-limbalus, angulo utroque in lobulum nigrum producto. - Long. corp. $2 \frac{5}{\frac{5}{2}}-2 \frac{1}{12}$ lin., Long. al. $3 \frac{1}{6}-3 \frac{5}{12}$ lin.

Syn. Empis punctata Meig. Klass. 227. 22.

Empis punctata Meig. Syst. Beschr. III. 38. 41. 
Empis ignota Mleig. Syst. Beschr. VI. 338.

Empis ignota Z ett. Dipt. Scand. I. 380. 13.

Hab. Europa fere tota.

7. Emp. semicinerea m. $\delta$ et $ᄋ$. Cinerea, abdomine pedibusque flavis, antennis nigris, vittarum dorsalium interstitiis nigrofuscis. Hypopygii lamellae laterales oblusae, supera simplex, margine summo subfusco. - Long. corp. $2 \frac{5}{\frac{5}{2}}-3$ lin., Long. al. $3-$ $3 \frac{1}{3}$ lin.

Hab. Germaniae regiones montuosae.

8. Emp. trigramma Meig. ठ大 et +. Flava, antennis, macula permagnâ occipitis thoracisque vittis media et lateralibus nigris, interstitiis tamen villarum flavis. Hypopygii lamellae laterales subaculae; lamella supera simplex, majuscula, quasi turrita. - Long. corp. $2 \frac{5}{1 \frac{2}{2}}-2 \frac{3}{4}$ lin., Long. al. $3-4$ lin.

Syn. Empis trigramma Meig. Syst. Beschr. III. 38. 42.

Empis trigramma Zett. Dipt. Scand. I. 379. 12.

Hab. Europa media et septentrionalis.

9. Emp. lutea Meig. $\delta^{7}$ et ㅇ․ Flava, fronte concolore, antennis nigris. Hypopygii lamellae laterales in conum oblique descendentem, nigro-pilosum productae; lamella supera simplex. Long. corp. $1 \frac{1}{1} \frac{1}{2}-2 \frac{2}{1}$ lin., Long. al. $2 \frac{1}{1} \frac{1}{2}-2 \frac{2}{3}$ lin.

Syn. Empis lutea Meig. Syst. Beschr. III. 37. 40.

Hab. Europa media.

10. Emp. nana m. $\sigma^{\top}$ et 우. Flava, antennis nigris, fronte magnâ ex parte nigricante. In hypopygio summo appendices duae filiformes nigrae. - Long. corp. $1 \frac{7}{1 \frac{1}{2}}-2 \frac{1}{4}$ lin., Long. al. $2 \frac{6}{12}-$ $2 \frac{7}{12}$ lin.

Hab. Styria et Carinthia.

11. Emp. parvula Egg. ठ et 오. Flava, tertio antennarum articulo, linể mediâ thoracis tenui et haud raro obsoletâ, stigmale denique prothoracis nigris, primis duobus antennarum articulis sub. aequalibus. - Long. corp. $2 \frac{1}{4}-2 \frac{7}{12}$ lin., Long. al. $3 \frac{1}{4}-3 \frac{5}{2}$ lin.

Syn. Empis parvula Egg. Verh. d. zool. bot. Ver. X. 333.

Hab. Germania australis. 
Anhangsweise will ich hier noch zwei Arten besprechen, welche sich durch ihre gelbe Körperfärbung dem Verwandtschaftskreise der Emp. stercorea anzuschliefsen scheinen könnten, die aber in ihrem Körperbaue viel zu sehr abweichen, als dafs sie zu demselben gezogen werden dürften.

Die erste der beiden Arten ist die von Scholtz in der Schlesischen entomologischen Zeitung V. p. 53 beschriebene Emp. dis par, welche sich durch die dichte, schneeweifse Bestäubung des Männchens sehr auszeichnet. Hinsichtlich der Unbehaartheit der Mittellinie des Thorax und hinsichtlich der Schlankheit und Kahlheit der Beine stimmt sie mit den in den Verwandtschaftskreis der Emp. slercorea gehörigen Arten überein. Auch in der Körperform, sowie in der Gestalt und Aderung der Flügel weicht sie von denselben nicht wesentlich ab. Der Bau der Fühler ist dem der Empis parvula ähnlich, würde also auch noch keinen Grund geben, sie nicht in jenen Verwandtschaftskreis zu stellen, was zu thun aber durch die runde Gestalt des Kopfes und durch die auf der Stirn zusammenstofsenden Augen des Männchens auf das Bestimmteste verboten wird.

Die zweite Art nenne ich Emp. mesogramma. Sie hat aus. ser der rothgelben Färbung in der That nicht viel mit den Arten aus dem Verwandtschaftskreise der Emp. stercorea gemein. Ihr Körperbau ist viel robuster, sie hat kürzere und mehr behaarte Beine, sowie verhältnifsmäfsig kürzere und breitere Flügel; der Bau der Fühler ähnelt dem der Emp. parvula und dispar, doch ist das erste Glied derselben verhältnifsmäfsig etwas länger; der Kopf ist rund und die Augen des Männchens stofsen auf der Stirn vollkommen zusammen; die Behaarung des Thorax ist länger und auch auf der ganzen Mittellinie desselben stehen lange borstenartige Haare. Der ganze Körper ist rothgelb gefärbt. Dieselbe Farbe haben die beiden ersten Fühlerglieder, während das dritte Glied mit Ausnahme der Wurzel schwarz gefärbt ist. Die Stirn und der gröfste Theil des Hinterkopfs sind schwarz, erstere bei dem Weibchen weifslich bestäubt. Der ziemlich dicke Rüssel reicht bis nahe an die Spitze der Vorderhüften. Die Oberseite des Thorax hat ein auffallend mattes Ansehen und ist von dünner weifser Besläubung bedeckt, welche aber auf den Interstitien zwischen Mittelstrieme und Seitenstriemen fehlt, so dafs diese ein wenig dunkler erscheinen; die bis ganz vorn hin stark behaarte Mittellinie ist mattschwarz gefärbt. Prothoraxstigma und Schildchen sind mit dem übrigen Tho- 
rax gleichgefärbt; letzteres trägt am Rande vier bis sechs schwarze Borsten. Der Hinterrücken ist rostbraun oder schwarzbraun gefärbt. Der Hinterleib ist entweder ganz und gar rothgelb, oder hat auf seiner Milte eine Längsreihe undeutlich begrenzter schwarzbrauner Flecke, welche mehr oder weniger zu einer Strieme zusammenfliefsen; dunkle Seitenstriemen sind nicht vorhanden. Das gelbe Hypopygium ist ziemlich klein; die seitlichen Lamellen desselben sind ziemlich schmal, erweitern sich auf der Mitte ihrer Oberseite, werden dann wieder schmäler und endigen fast löffelförmig; das Ende derselben ist tiefschwarz gefärbt und mit langen schwarzen Haaren besetzt; die obere Lamelle ist an ihrem Oberrande geschwärzt und sendet von der Mitte desselben zwei tiefschwarze, zusammen ein $\mathbf{V}$ bildende Anhänge aus; der gelbe Penis bildet in der Nähe seiner Basis eine Art von auffallenden Knoten und ist von einer ziemlich ansehnlichen, zungenförmigen gelben Lamelle unterstützt. Beine gelb; das zweite Hüftglied und die äus. serste Spiłze der Schenkel mit den gewöhnlichen, kleinen schwarzen Makeln; die Füfse fast von der Wurzel an stark gebräunt; die Behaarung derselben schwarz und bei dem Männchen viel länger als bei dem Weibchen. Bei dem Männchen sind auf der Unterseite die Vordersehenkel kahl, die Mittelschenkel mit ziemlich langen aber nicht sehr zahlreichen, die Hinterschenkel nur mit etlichen ganz vereinzelten borstenartigen Haaren besetzt; die Behaarung seiner Vorderschienen ist nicht lang, dagegen sind die Mittelschienen mit auffallend langen, borstenartigen Haaren besetzt, welche auf der Unterseite derselben ziemlich dicht stehen, und auch auf der Oberseite der Hinterschienen finden sich lange, doch wenig zahlreiche, borstenartige Haare; die Behaarung der Füfse ist ziemlich lang, namentlich an dem Ende der einzelnen Fufsglieder. Das erste Glied der Vorderfüfse des Männchens ist nicht verdickt. Die Flügel haben eine deutliche, lehmbräunliche Trübung und lehmbräunliche, in der Nähe der Flügelwurzel mehr blafs lehmgelbliche Adern; ein deutliches Randmal ist nicht vorhanden. Das Flügelgelgeäder ist vollständig, die Discoidalzelle am Ende ziemlich schief abgeschnitten. Länge des Körpers $3 \frac{1}{4}-3 \frac{1}{2}$ Lin., Flügellänge $3 \frac{1}{2}-4$ Lin.

\section{Vaterland: Schlesien.}

Ich bin lange zweifelhaft gewesen, ob gegenwärtige Art nicht Meigen's Emp. monogramma sein könne. Da Meigen dieselbe ausdrücklich ,glänzend rothgelb" nennt, meine Art sich aber gerade 
durch ihre auffallend glanzlose Färbung auszeichnet, da ferner die Oberseite des Hinterleibs der Meigen'schen Art schwarzbraun und nur die Unterseite desselben gelb sein soll, während bei meiner Art entweder der ganze Hinterleib gelb oder doch nur mit einer Reihe verwischter schwarzbrauner Mittelflecke gezeichnet ist, scheint es mir viel zu gewagt, meine Art für Emp. monogramma Meig. zu erklären. -

Dafs Emp. mesogramma in den Verwandtschaftskreis der Emp. nigricans Meig. gehört, ist nicht zu verkennen. Die zusammenslofsenden Augen des Männchens, die Stärke der Oberlippe, die Behaartheit der Interstitien bei der Nacktheit der Thoraxstriemen, das vollständige Flügelgeäder mit der am Ende schief abgeschnittewen Discoidalzelle, die verhältnifsmäfsig ansehnliche Länge der Beine und ihre ziemlich schwache und verhältnifsmäfsig sparsame Beborstung, die langen zwar nicbt verdickten, aber doch plumpen Füfse, so wie das gerade ausgestreckte Hypopygium mit seinen langen, schmalen, seitlichen und mit seiner sehr entwickelten oberen Lamelle sind die hauptsächlichsten diese Gruppe charakterisirenden Merkmale. Von den mir bekannten europäischen Empis-Arten gehören 13 zu derselben. Die durch mehr oder weniger gefleckte Flügel ausgezeichneten Arten sind: 1) variegata Meig., 2) affinis Egg., 3) confusa Lw., 4) maculata Fbr., 5) apicalis Lw. und 6) rava Lw. - Ihnen schliefst sich sehr nahe 7) macra nov. sp. aus Griechenland an, welche der Enıp. apicalis sehr ähnlich ist, sich aber durch etwas abweichenden Bau des Hypogygiums und durch völligen Mangel dunklerer Färbung an der Flügelspitze unterscheidet. Die übrigen Arten sind: 8) meridionalis Meig., 9) nigricans Meig., 10) cognata Egg., 11) discolor Loew, 12) alpina nov. sp., 13) mesogramma Lw. - Die in der Schweiz lebende Empis alpina, von der ich nur das Weibchen besitze, hat die Gröfse der Empis discolor, aber einen etwas robusteren Körperbau; sie ist ganz gelb, nur der Thoraxrücken mit Ausnahme der Schultern und des Seitenrands gelb; der Hinterleib hat eine schmale braunschwarze Mittelstrieme; das dritte Fühlerglied schwarz; die Füfse bis gegen die Basis hin braunschwarz; die Flügel wie bei Emp. discolor. - 


\section{$2 \mathrm{BHL}$ Biodiversity Heritage Library}

Loew, H. 1867. "Ueber den Verwandtschaftskreis der Empis stercorea Lin." Berliner entomologische Zeitschrift / herausgegeben von dem Entomologischen Vereine in Berlin 11(102),11-24. https://doi.org/10.1002/mmnd.18670110103.

View This Item Online: https://www.biodiversitylibrary.org/item/36408

DOI: https://doi.org/10.1002/mmnd.18670110103

Permalink: https://www.biodiversitylibrary.org/partpdf/210189

\section{Holding Institution}

Smithsonian Libraries

\section{Sponsored by}

Smithsonian

\section{Copyright \& Reuse}

Copyright Status: Public domain. The BHL considers that this work is no longer under copyright protection.

This document was created from content at the Biodiversity Heritage Library, the world's largest open access digital library for biodiversity literature and archives. Visit BHL at https://www.biodiversitylibrary.org. 